\title{
The impact of sexual activity on serum hormone levels after penile prosthesis implantation
}

\author{
Onder Canguven, Raidh A. Talib, Ahmed Shamsodini, Abdulla Al Ansari \\ Hamad General Hospital, Urology Department, Doha, Qatar.
}

\begin{abstract}
Summary Objectives: Penile prosthesis implantation is the final treatment option for patients who have erectile dysfunction. Most of the patients use their penile prosthesis successfully and frequently for penile-vaginal intercourse. Previous literature showed that decrease in sexual activity resulted in decreased serum testosterone levels and vice versa. The aim of this study was to examine the impact of sexual activity on serum sex hormone levels after penile prosthesis usage.

Material and methods: In this study, we examined sixtyseven patients for their sex hormone changes who had penile prosthesis surgery $2.7 \pm 1.5$ years ago.

Results: Patients were using their penile prosthesis for sexual activity with a mean of $9.9 \pm 5.7$ times per month. Dehydroepiandrosterone sulfate was significantly higher compared to pre-surgery results (5.3 \pm 2.6 vs $4.5 \pm 2.9$; $p=0.031$ ). Mean serum total testosterone levels of patients before and after penile prosthesis usage were clinically significant $15.78 \pm 4.8 \mathrm{nmol} / \mathrm{L}$ and $16.5 \pm 6.1$ $\mathrm{nmol} / \mathrm{L}$, respectively. Mean serum luteinizing hormone levels of patients before and after penile prosthesis usage were $3.98 \pm 2.16 \mathrm{IU} / \mathrm{L}$ and $5.47 \pm 4.76 \mathrm{IU} / \mathrm{L}$, respectively. No statistical significance difference was observed in the mean total and free testosterone, estradiol and luteinizing hormone levels between pre- and post-surgery. Conclusion: This study results demonstrated that sexual activity changed sex hormone levels positively among those men who were implanted penile prosthesis because of erectile dysfunction.
\end{abstract}

KEY WORDS: Androgens; Erectile dysfunction; Hypogonadism; Prosthesis; Testosterone.

Submitted 9 April 2014; Accepted 30 June 2014

\footnotetext{
INTRODUCTION

Erectile dysfunction (ED) is defined as the inability to achieve or maintain an erection sufficient for satisfactory sexual intercourse. Many of patients with ED have not had a normal erection in many months or years. Previous studies demonstrated that sexual inactivity results in reversible reduction of luteinizing hormone (LH) and serum testosterone levels in ED patients compared to
}

healthy men $(1,2)$. Testosterone, which has androgenic and anabolic effects on human body, decline gradually with aging in males. Major benefits of testosterone on sexual function, mood, and strength are well known from the ancient times. Currently, evidence supports the concept that normal testosterone levels reduce cardiovascular disease risk, decrease fat, decrease total cholesterol, increase muscular body mass, and display good glycemic control (3). Moreover, epidemiological studies imply that many important disease states and related comorbidities are linked to low testosterone levels (4).

Although there are mixed results from animal studies (5), male testosterone concentrations before and after sexual activity were shown as increased in human studies $(6,7)$. Insertion of a penile prosthesis (PP) for men who cannot use or fail to respond to first and second line treatments is the final treatment option for ED. Actually PP provides a satisfactory, definitive solution for ED. In different patient satisfaction studies it was shown that, of the PP implanted men more than 90\% stated they were still using the PP for sexual intercourse with an average frequency of coitus of 5 times monthly (8-10). A recent study conducted by Escasa et al. supported previous studies and confirmed that sexual activity increases testosterone level which was more prominent in participants who had sexual intercourse (7). The aim of this study is to examine the influence of sexual activity on serum sex hormone levels after PP practice.

\section{MATERIALS AND METHODS}

After institutional review board approved the study, a retrospective chart analysis was performed on patients that underwent PP implantation surgery from January 2009 through January 2013. All participants provided written informed consent. Enrollment and data collection were conducted at follow-up visits at least 6 months and up to 4 years after implantation.

The 67 patients with PP implantation reported in this study were ambulatory patients seen at our andrology clinic who met all of the following criteria. The principal eligibility criteria included patients who were requested hormonal profile examined in this study (total and free testosterone, estradiol, dehydroepiandrosterone sulfate (DHEAS) and LH) in the last month before the surgery 
they had. Another important inclusion criteria for the participation was regular sexual intercourse with his wife after PP implantation surgery. Prostate cancer, creatinine $>2$ $\mathrm{mg} / \mathrm{dL}$, myocardial infarction or stroke within 6 months, or congestive heart failure, and use of androgens, antiandrogens comprised major exclusion criteria. Medications that influence hormone levels, such as high-dose opiates, glucocorticoids, antiepileptics or any kind of herbal drugs also excluded participation. Fasting blood samples were obtained between 7-9 AM in the morning. Testosterone, LH, DHEAS and estradiol levels were measured by standard RIA kits. We also asked Global Satisfaction Question (GSQ) "Did the implant permit you to experience satisfactory sexual relations?" and a frequency question "How many times do you use your PP for sexual activity each month?" to all participants at the time of their participations.

\section{Statistical analysis}

Qualitative and quantitative data values were expressed as frequency (percentage) and mean \pm SD. Quantitative variables means between pre and post surgery groups were compared using paired t-test. Pearson correlation coefficient was used to examine and assess the linear relationship between the two quantitative variables. Pictorial presentations of the key results were made using appropriate statistical graphs. A two-sided $\mathrm{P}$ value $<0.05$ was considered to be statistically significant. All statistical analyses were done using statistical packages SPSS 19.0 (SPSS Inc. Chicago, IL).

\section{Results}

Sixty-seven male subjects who had active sexual life with aid of PP were enrolled in the study. Mean age of patients was $59.9 \pm 10.9$ (range: $30-82$ ) years. The mean duration of ED problem was $2.7 \pm 1.5$ years. With the GSQ, sixtyseven patients who responded as 'yes' were included to our study group. With the frequency question, we aimed to learn frequency of the penile intercourse: 64 patients (95.5\%) responded to use it frequently, while three (4.5\%) declared they use it rarely because of partner related problems. The average of PP usage for sexual activity was $9.9 \pm 5.7$ (range: 2 -28) times per month. The mean time from surgery was 22.6 months (range: 6 months-48 months). Paired t-test revealed that post-surgery mean DHEAS was significantly higher compared pre-surgery results $(5.3 \pm 2.6$ vs $4.5 \pm 2.9 \mu \mathrm{mol} / \mathrm{L} ; \mathrm{p}=0.031$ ) (Figure $1)$. No statistical significance difference was observed in the mean total and free testosterone, estradiol and LH between pre- and post-surgery results. Mean values following post-surgery was found to be observationally higher compared to pre-surgery group for total testosterone $(16.5 \pm 6.1 \mathrm{vs} 15.8 \pm 4.8 \mathrm{nmol} / \mathrm{L} ; \mathrm{p}=0.195)$, free testosterone $(86.7 \pm 15.9$ vs $79.7 \pm 20.4 \mathrm{pmol} / \mathrm{L} \mathrm{p}=$ $0.258)$, estradiol $(11.67 \pm 4.69 \mathrm{vs} 10.58 \pm 3.79 \mathrm{pmol} / \mathrm{dL}$; $\mathrm{p}=0.191)$, and $\mathrm{LH}(5.1 \pm 4.5$ vs $4.2 \pm 2.2 \mathrm{IU} / \mathrm{L}) ; \mathrm{p}=$ $0.158)$, however these differences were statistically insignificant ( $p>0.05)$. Further, Pearson's correlation analysis revealed that variable monthly intercourse were inversely or negatively related to estradiol, LH and age (correlation coefficient $r<-0.3$ ) again this correlation coefficient values were not statistically significant ( $p>0.05)$.
Figure 1.

Serum values of total testosterone, Luteinizing Hormone ( $L H)$, Estradiol and Dehydroepiandrosterone sulphate (DHEAS) levels. Values are expressed as means of 67 patients before and after usage of penile prosthesis. * $p<0.05$

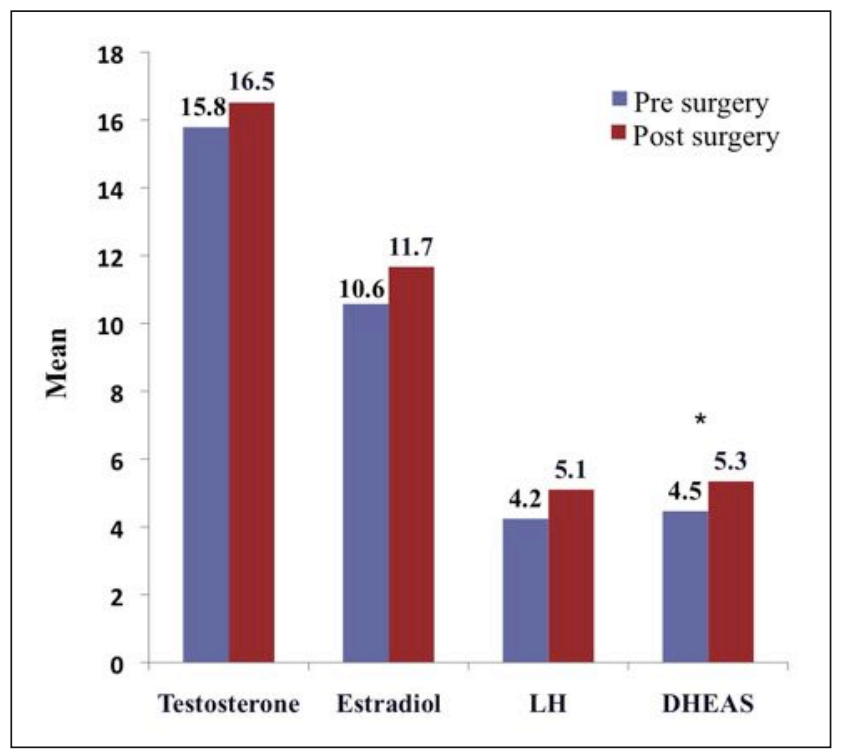

\section{Discussion}

We tested whether PP usage after a long period of time having no penile-vaginal intercourse and sexual activity in ED patients' impact on sex hormone levels. We showed that PP usage caused a significant increase in DHEAS levels after PP usage. We also demonstrated that mean total and free testosterone, estradiol, LH levels were clinically increased after PP usage, however the results were statistically insignificant. Our results carry important clinical implications. In particular, the findings suggest that PP implantation presents not only a change in sex life of a man but also change in hormone levels in a positive direction. These increases, especially, becomes more important when we take longitudinal studies into account. Because, longitudinal studies in male aging researches have demonstrated that serum testosterone levels decline with age (11).

More than forty years ago, a motivating observational study published in journal Nature and provided some clues about the effects of sexual activity on testosterone (12). A lone man on an island noted that his beard appeared to grow more around the time of trips to the mainland. As beard growth could be accepted as an indirect sign for testosterone levels, he hypothesized that the sexual interest and activity increased his testosterone, which in turn increased his beard growth (12).

In forthcoming years, researchers found that androgens and estrogens were significantly higher following masturbation, but interestingly not after "sham masturbation" (13). In different studies, it was also proven that sexually explicit movies increase men's testosterone compared with sexually neutral films $(6,14)$. The development and easy use of salivary testosterone measurement has greatly simplified the inclusion of endocrine variables in biobehavioral research $(7,15)$. In one of these studies, it was demonstrated that testosterone increased in heterosexual 
men even exposed to brief conversations with women (15). Moreover, show off behaviors was associated with the increase in degree of testosterone. Assorted research results have indicated that testosterone increases can occur in a short period, often within $15 \mathrm{~min}$, in response to relevant stimuli, such as sexual activities or competitions $(7,16)$. In a sex club, researchers found that increase in men's testosterone level were more pronounced among those participating in sexual activity rather than observing (7). Additionally, men's testosterone changes were unrelated to their age (7).

According to previous studies, it is obvious that any sexual activity influences testosterone levels in a positive direction more or less $(7,13,16)$. In a previous study, Jannini et al found dramatic increase in serum total and free testosterone levels three months after various ED therapies including two PP implanted patients (1). Contrary to the latter study, although testosterone levels increased in our study, it was not significant. This could be due to the fact that the experimental setting in our study used evaluated testosterone levels not acutely or some weeks after treatment, but after years. The small population size (two PP implanted patients) in Jannini et al study might be another explanation for the dramatic increase in testosterone level (1).

Another study also demonstrated that married men have reduced morbidity and mortality compared with single men (17). On the other hand, Gray et al investigated a population of men and found that married men have parallel testosterone levels as single men, but that polygamously married men had higher testosterone than all other men (18). A variety of species have repeatedly been shown to respond to different female stimuli by triggering an increase in luteinizing hormone which was followed by a rise in plasma testosterone levels (19).

It is well known and proven that normal testosterone level is vital for general health $(3,20)$. Testosterone has been shown to produce positive effects on endothelial function, glucose metabolism, body composition and mood. Although the significance of testosterone is well known, the extent to which testosterone deficiency is involved in the pathogenesis of these conditions is unknown. Additionally, which type of testosterone formula could be useful in treatment of hypogonadism is an area of great interest and being searched. At the present time, it is recommended that androgen replacement should be taken in the form of natural testosterone.

The significance of hormone alterations related to sexual activity is not known and has not been studied directly. However, it is obvious that acute increase of testosterone should present some benefits to body during sexual intercourse or after it. Most studies investigated the effects of hormones on behavior, because hormones have powerful effects on the different body systems throughout life (21). On contrary, our behaviors also affect our hormones that were verified in different studies $(2,7)$. The "Challenge hypothesis" suggests that social interactions affect testosterone levels in males, and testosterone should be high when challenges for sources or the likelihood of challenges are present (22). If we accept the "challenge hypothesis" as true, there should be more in details of testosterone increase. Non-genomic actions of steroid hormones are those in which gene transcription is not directly implicated and involves second messenger participation and are rapid in action (within seconds to minutes) (23). Scientific information regarding the testosterone is not limited to its' genomic effect, it has also non-genomic effect $(23,24)$. Animal experiments and clinical studies showed rapid non-genomic relaxant effects of androgens on the smooth musculature of coronary arteries and the aorta $(25,26)$.

A possible protective effect of testosterone during sexual activity might be against skeletal muscle fatigue that was suggested earlier while investigating neuromuscular activity and hormonal profile in athletes (24). It is intriguing to speculate about the possible mechanisms linking testosterone and sexual activity.

Additionally, according to epidemiological studies there is a positive correlation between testosterone levels and mood (27). Therefore there may be different and important issues involved in the testosterone-sexual activity relation. Increase in testosterone and so on mood might be translated into increased confidence and behavior in difficult circumstances e.g. sexual activities. We propose, based on results of prior non-genomic testosterone research, that acute elevations in testosterone concentration may be able to reduce or compensate the effects of fatigue during sexual activities in addition to prepare the body for a successful intercourse. However, other possible explanations of increase in testosterone levels among men during sexual activity are warranted. In this study, we demonstrated significant increase in DHEAS levels after PP usage. Actually, DHEA has very low androgenic potency, but serves as the major direct or indirect precursor for most sex-steroids. DHEA is secreted by the adrenal gland and production is at least partly controlled by adrenocorticotropic hormone. The bulk of DHEA is secreted as a 3-sulfoconjugate i.e. DHEAS. In gonads and several other tissues, most notably skin, steroid sulfatases can convert DHEAS back to DHEA, which can then be metabolized to stronger androgens and to estrogens. Significant increase in DHEAS levels after PP usage may be due to utilize it as precursor of $\mathrm{T}$ in addition to testicular production. In our study, the testosterone increase was being driven from the higher levels. As has been demonstrated in the previous studies $(1,2,6)$, the current study showed that sexual activity influence serum testosterone levels by increase in LH secretion in males. In a recent review article, researchers looked for potential health benefits of various sexual activities and focused on the effects of different sexual activities (28). Among different sexual activities, penile-vaginal intercourse was shown associated with better psychological and physiological health indices (28). From a practical standpoint, our findings imply that penile-vaginal intercourse augmented by PP improved our patients' testosterone level that is crucial for general health. Despite its practical value, our study has limitations. Firstly, it consists of lack of prospective study design. Secondly, the difference in the time interval between surgery and blood taken for controlling testosterone levels were not same. Thirdly, sample size was relatively small. This was mainly due to reluctance of our PP patients to participate in this study that was done years after the surgery. Since they did not have any problem and satisfied 
with their PP, they did not want to participate and give blood samples. According to literature, androgen levels have circadian rhythm and exhibit daily and seasonal fluctuations in addition to age-related decline (21). Unfortunately, we did not classify our study patients' testosterone levels according to their season when blood samples given. However, all blood samples after PP usage were taken in the same month from all participants.

\section{Conclusions}

Studies, especially in the last decades, demonstrated that sex hormones are closely related with health risks and promotes health. Our study investigated possible correlations of the sex hormone levels and sexual intercourse in patients with PP. In conclusion, the present study suggests that penile-vaginal intercourse by PP usage appears to be increasing sex hormone levels relative to baseline levels that were before the PP implantation. However, further prospective controlled studies with large sample size are needed to determine why and how PP usage leads to change in sex hormone levels and how long this alteration continues.

\section{ETHICAL STANDARDS}

Written informed consent was obtained from patients who participated in this study.

\section{ACKNOWLEDGEMENTS}

A grant from the Hamad Medical Corporation primarily supported this research. We would also like to acknowledge the careful work of Dr. Prem Chandra for his assistance with the statistics used in this study.

\section{References}

1. Jannini EA, Screponi E, Carosa E, et al. Lack of sexual activity from erectile dysfunction is associated with a reversible reduction in serum testosterone. Int J Androl. 1999; 22:385-92.

2. Carosa E, Benvenga S, Trimarchi F, et al. Sexual inactivity results in reversible reduction of LH bioavailability. Int J Impot Res. 2002; 14:939; discussion 100

3. Traish AM, Saad F, Feeley RJ, Guay A. The dark side of testosterone deficiency: III. Cardiovascular disease. J Androl. 2009; 30:477-94.

4. Stanworth RD, Jones TH. Testosterone for the aging male; current evidence and recommended practice. Clin Interv Aging. 2008; 3:25-44.

5. Hilliard J, Pang CN, Penardi R, Sawyer CH. Effect of coitus on serum levels of testosterone and LH in male and female rabbits. Proc Soc Exp Biol Med. 1975; 149:1010-4.

6. Stoleru SG, Ennaji A, Cournot A, Spira A. LH pulsatile secretion and testosterone blood levels are influenced by sexual arousal in human males. Psychoneuroendocrinology 1993; 18:205-18.

7. Escasa MJ, Casey JF, Gray PB. Salivary testosterone levels in men at a U.S. sex club. Arch Sex Behav. 2011; 40:921-6.

8. Lux M, Reyes-Vallejo L, Morgentaler A, Levine LA. Outcomes and satisfaction rates for the redesigned 2-piece penile prosthesis. J Urol. 2007; 177:262-6.

9. Carson CC, Mulcahy JJ, Govier FE. Efficacy, safety and patient satisfaction outcomes of the AMS $700 \mathrm{CX}$ inflatable penile prosthesis: results of a long-term multicenter study. AMS 700CX Study Group. J Urol. 2000; 164:376-80.
10. Bettocchi C, Palumbo F, Spilotros M, et al. Patient and partner satisfaction after AMS inflatable penile prosthesis implant. J Sex Med. 2010; 7:304-9.

11. Harman SM, Metter EJ, Tobin JD, et al. Longitudinal effects of aging on serum total and free testosterone levels in healthy men. Baltimore Longitudinal Study of Aging. J Clin Endocrinol Metab. 2001; 86:724-31.

12. Anon. Effects of sexual activity on beard growth in man. Nature. 1970; 226:869-70.

13. Purvis K, Landgren BM, Cekan Z, Diczfalusy E. Endocrine effects of masturbation in men. J Endocrinol. 1976; 70:439-44.

14. Pirke KM, Kockott G, Dittmar F. Psychosexual stimulation and plasma testosterone in man. Arch Sex Behav. 1974; 3:577-84

15. Roney JR, Lukaszewski AW, Simmons ZL. Rapid endocrine responses of young men to social interactions with young women. Horm Behav. $2007 ; 52: 326-33$

16. Dabbs Jr JM, Mohammed S. Male and female salivary testosterone concentrations before and after sexual activity. Physiology \& Behavior. 1992; 52:195-7.

17. Hu YR, Goldman N. Mortality differentials by marital status: an international comparison. Demography. 1990; 27:233-50.

18. Gray PB. Marriage, parenting, and testosterone variation among Kenyan Swahili men. Am J Phys Anthropol. 2003; 122:279-86.

19. Gleason ED, Fuxjager MJ, Oyegbile TO, Marler CA. Testosterone release and social context: when it occurs and why. Front Neuroendocrinol. 2009; 30:460-9.

20. Laughlin GA, Barrett-Connor E, Bergstrom J. Low serum testosterone and mortality in older men. J Clin Endocrinol Metab. 2008; 93:68-75.

21. Anders S, Watson N. Social neuroendocrinology: Effects of social contexts and behaviors on sex steroids in humans. Human Nature. $2006 ; 17: 212-37$

22. Wingfield JC, Hegner RE, Dufty Jr. AM, Ball GF. The 'challenge hypothesis': Theoretical implications for patterns of testosterone secretion, mating systems and breeding strategies. The American Naturalist. 1990; 136:829-46.

23. Waldkirch E, Uckert S, Schultheiss D, et al. Non-genomic effects of androgens on isolated human vascular and nonvascular penile erectile tissue. BJU international. 2008; 101:71-5; discussion 5.

24. Bosco C, Colli R, Bonomi R, et al. Monitoring strength training: neuromuscular and hormonal profile. Med Sci Sports Exerc. 2000; 32:202-8.

25. Yue P, Chatterjee K, Beale C, et al. Testosterone relaxes rabbit coronary arteries and aorta. Circulation. 1995; 91:1154-60.

26. Deenadayalu VP, White RE, Stallone JN, et al. Testosterone relaxes coronary arteries by opening the large-conductance, calcium-activated potassium channel. Am J Physiol Heart Circ Physiol. 2001; 281:H1720-7.

27. Barrett-Connor E, Von Muhlen DG, Kritz-Silverstein D. Bioavailable testosterone and depressed mood in older men: the Rancho Bernardo Study. J Clin Endocrinol Metab. 1999; 84:573-7.

28. Brody S. The Relative Health Benefits of Different Sexual Activities. J Sex Med. 2010; 7:1336-61.

\section{Correspondence}

Onder Canguven, MD

ocanguven@yahoo.com

Raidh A. Talib, MD

Ahmed Shamsodini, MD

Abdulla Al Ansari, MD

Hamad General Hospital Urology Department - 3050, Doha, Qatar 